\title{
La arquitectura popular como modelo de edificación sostenible. El ejemplo de Tierra de Campos
}

\author{
Juan CORTÉs PEDROSA \\ Juan.cortes.pedrosa@gmail.com
}

Recibido: 18 de septiembre de 2013

Enviado a evaluar: 23 de septiembre de 2013

Aceptado: 3 de octubre de 2013

\section{RESUMEN}

En el presente estudio, se pretende establecer las relaciones de causa-efecto entre las características de la arquitectura popular de la región de Tierra de Campos, condicionadas por su situación geográfica, ecológica y social, y el paisaje tradicional, que resulta de la combinación de estos elementos con el resto del medio físico. También se busca valorar la importancia del hábitat, formado mayoritariamente por conjuntos de arquitectura popular de dicho territorio, como elemento del paisaje y en relación con el resto de elementos, como la trama parcelaria, los usos del suelo y la red fluvial y de infraestructuras.

Palabras clave: Arquitectura popular, edificación sostenible, paisaje, medio ambiente.

\section{The architecture as a model of sustainable building. The example of Tierra de Campos.}

\begin{abstract}
In the present study aims to establish the cause-effect relationships between the characteristics of the architecture of the region of Tierra de Campos, conditioned by its geographical, ecological and social, and cultural landscape, which is a combination of these elements with the rest of the physical environment. It also seeks to assess the importance of habitat, made up mostly of popular architectural ensembles that territory, as part of the landscape and in relation to the other elements, such as plot land consolidation, land use and river network and infrastructure.
\end{abstract}

Key words: Architecture, sustainable building, landscape, environment.

\section{RÉSUMÉ}

L'architecture comme un modèle de construction durable. L'exemple de la Tierra de Campos.

Dans la présente étude vise à établir les relations de cause à effet entre les caractéristiques de l'architecture de la région de Tierra de Campos, conditionnée par son paysage géographique, écologique et sociale, et culturelle, qui est une combinaison de ces éléments avec le reste de l'environnement physique. Il vise également à évaluer l'importance de l'habitat, essentiellement composée d'ensembles architecturaux populaires ce territoire, dans le cadre du paysage et en relation avec les autres éléments, tels que le remembrement des parcelles, l'utilisation des terres et le réseau fluvial et les infrastructures.

Mots-clés: Architecture, construction durable, le paysage, l'environnement. 


\section{INTRODUCCIÓN}

Los impactos ambientales negativos producidos por el sector de la construcción adquieren una gran relevancia dentro del actual contexto de crecimiento de la población mundial, que demanda un gran esfuerzo de desarrollo urbanístico por parte de los países denominados como potencias emergentes ${ }^{1}$. La experiencia vivida en los países occidentales a partir del final de la Segunda Guerra Mundial, que originó una gran demanda de alojamiento rápido y económico, resultó mayoritariamente insatisfactoria desde el punto de vista ambiental y estuvo marcada por la enorme difusión del modelo edificatorio residencial promovido por el Movimiento Moderno ${ }^{2}$. Esta tendencia arquitectónica aprovechó al máximo las nuevas herramientas tecnológicas disponibles, como los materiales de gran resistencia estructural -el hormigón armado y el acero- o las instalaciones de acondicionamiento térmico. Todo ello fue posible gracias al enorme aporte energético conseguido a través de la quema de combustibles fósiles, sin tener en cuenta posibles externalidades negativas, como la contaminación de la atmósfera.

A su vez, se desestimaron o se olvidaron inconscientemente los conocimientos provenientes de las tradiciones constructivas locales, en beneficio de una forma de producción estandarizada, que se extendió por todos los ámbitos geográficos, independientemente de sus condiciones ambientales, como el microclima local o la vegetación existente. Las consecuencias de este fenómeno se siguen sufriendo hoy en día $y$, entre otras, se pueden citar el despilfarro energético, las emisiones de gases de efecto invernadero, la destrucción de numerosos ecosistemas, la reducción de la biodiversidad y la degradación del paisaje tradicional. La intención de revertir o, al menos, paliar estos procesos, que disminuyen la calidad del entorno del ser humano y ponen en serio peligro el desarrollo económico y social, hacen volver la mirada hacia otros modelos edificatorios, menos dañinos desde el punto de vista ambiental.

En este contexto, surge un renovado interés por la arquitectura popular, como modelo de hábitat integrado en la naturaleza, que puede proporcionar ciertas claves para restablecer una relación equilibrada entre las necesidades del ser humano y las condiciones ecológicas del medio. Esta concepción de la naturaleza como fuente de recursos finitos, que no deben ser explotados de forma indiscriminada hasta su agotamiento, se recupera en alguna medida a través del desarrollo sostenible, que viene a superar los paradigmas de crecimiento indefinido, generalizados a partir de la Revolución Industrial (Sotelo, J. A., 2000). Una visión de la arquitectura popular como un micro-cosmos autosuficiente y "sustentable", que contribuye a mantener la estabilidad ecológica del entorno gracias a una inteligente explotación de los recursos dispo-

\footnotetext{
${ }^{1}$ En este aspecto se trataría, sobre todo, de Brasil, India y China.

${ }^{2}$ Se entiende por Movimiento Moderno el fenómeno arquitectónico que se extiende a partir de los años 20 del pasado siglo, liderado por autores como Le Corbusier y Mies Van der Rohe. Algunos críticos lo denominan también Estilo Internacional y se basa principalmente en el uso de una tipología residencial en bloque lineal abierto, rodeada de zonas verdes.
} 
nibles, sin dejar de lado el bienestar de sus habitantes, resulta enormemente atractiva dentro de un panorama de replanteamiento del actual modelo de desarrollo, basado en la producción ilimitada de bienes de consumo.

\section{DEFINICIÓN Y CARACTERÍSTICAS DE LA ARQUITECTURA POPULAR}

Antes de abordar la cuestión de la sostenibilidad es necesario definir el concepto de "arquitectura popular" que se va a emplear, ya que no existe unanimidad entre los principales autores en este campo, por lo cual el término se presta a cierta confusión con otros similares como arquitectura vernácula o tradicional, que tienen aspectos en común, pero conservando matices distintos ${ }^{3}$.

Teniendo en cuenta que la arquitectura es una disciplina claramente definida, al menos a partir del tratado De Architectura de Marco Vitrubio (Siglo I a.C.), el adjetivo "popular", que se le añade, significa "perteneciente o relativo al pueblo" o "que es peculiar del pueblo o procede de él" según las dos primeras acepciones del diccionario de la "Real Academia de la Lengua". Esto coincide con la definición de Carlos Flores, que sostiene que la arquitectura popular es "el arte y la técnica de proyectar, construir y transformar el entorno vital de este grupo social que hemos llamado el pueblo, realizándose todo ello... por individuos salidos del propio grupo". Si hubiera que explicar el concepto de forma coloquial se podría decir que se trata de la arquitectura del pueblo, para el pueblo y por el pueblo (Oliver, P., 1997), siendo especialmente significativo el último de estos aspectos, referido a la autoría no profesional de la misma. La autoconstrucción viene a suponer su verdadera cualidad diferencial y la dota de una autenticidad y funcionalidad que la separan nítidamente de lo que se viene a denominar como "arquitectura culta".

Del anteriormente referido carácter popular, y conjuntamente con su origen asociado al medio rural, se derivan cuatro componentes principales que definen este tipo de arquitectura, desde un punto de vista ecológico:

a) una gran dependencia del medio físico, causada por el escaso desarrollo tecnológico de las comunidades agrícolas pre-industriales,

b) el empleo de técnicas constructivas tradicionales, que garantizan la eficacia y durabilidad de sus soluciones,

\footnotetext{
${ }^{3}$ La arquitectura vernácula destaca su carácter doméstico o nativo, mientras que la arquitectura tradicional incluye otras realizaciones, además de las edificaciones populares, siempre que su factura se pueda encuadrar dentro de las tradiciones constructivas locales.

${ }^{4}$ También denominada arquitectura histórico-artística, incluye todos aquellos edificios representativos, que se suelen estudiar desde una perspectiva diacrónica, destacando la noción de estilo, asociada a una determinada etapa histórica.
} 
c) la repetición de unos modelos edificatorios atemporales, llamados tipos arquitectónicos,

d) la máxima adaptación funcional a las necesidades vitales y productivas de sus habitantes.

Un breve análisis de estas características muestra una clara correspondencia con las cuatro dimensiones de la sostenibilidad ${ }^{5}$, en efecto: la relación altamente dependiente del entorno provoca una actitud de respeto y cuidado hacia el medio ambiente; la factura primitiva de los edificios responde, sobre todo, a restricciones de tipo económico, en su sentido más amplio; el tipo genera una identidad cultural definida, asociada al territorio; y el modelo urbano adoptado refleja fielmente la organización social del correspondiente grupo humano. Se pueden definir, por lo tanto, cuatro enfoques complementarios:

1. La perspectiva medioambiental: resulta de gran interés el estudio de la arquitectura popular desde este punto de vista, se trata de una adaptación ecológica a las condiciones ambientales, ya que los recursos naturales disponibles en las comunidades rurales tradicionales (materiales de extracción, biomasa, agua, etc.) eran por lo general escasos y/o difíciles de explotar, por lo cual debían ser aprovechados de forma cuidadosa para no comprometer su disponibilidad a lo largo del tiempo.

2. La perspectiva económica: la edificación popular es una respuesta altamente eficiente a los requerimientos espaciales y funcionales de sus habitantes, mediante unos sistemas estructurales, constructivos y energéticos que, perfeccionados sucesivamente generación a generación, permiten alcanzar las necesarias condiciones de habitabilidad con el menor coste económico asociado.

3. La perspectiva cultural: se puede analizar desde dos enfoques distintos: estas construcciones son, junto con el terrazgo, el elemento más significativo del paisaje cultural y, por otra parte, forman un patrimonio etnográfico valioso, que simboliza la identidad de un territorio, mediante sus tipos arquitectónicos.

4. La perspectiva social: la arquitectura popular se puede entender también como un modelo de ocupación del territorio que, cumpliendo con la función de permitir la explotación productiva de los recursos agrarios, logra alcanzar también una gran cohesión social, generando los espacios públicos, como plazas y calles, que son necesarios para la vida en común.

A continuación, se desarrollarán estas cuatro dimensiones sostenibles de la arquitectura popular a través del caso concreto de la comarca vallisoletana de Tierra de

\footnotetext{
${ }^{5}$ A partir de los años 80 se extiende una tendencia, según la cual existen tres dimensiones principales de la sostenibilidad: medioambiental, económica y social. En el documento Culture: Fourth Pillar of Sustainable Development, publicado en 2010 por la Red Mundial de Ciudades, Gobiernos Locales y Regionales, se propone la inclusión de una cuarta dimensión, la cultura.
} 
Campos. El interés de este territorio proviene de la gran integración de su hábitat tradicional en el entorno, reflejando la adaptación de sus pobladores al medio físico en que se encuentran. También destaca por un paisaje ciertamente singular e inconfundible, que forma una unidad morfológica distintiva dentro del contexto regional de Castilla y León (García Merino, L. V., 1988). El débil modelado de su suavemente ondulante relieve, la homogeneidad de sus suelos arcillosos, el escaso caudal de unos cauces proclives a secarse y la falta de vegetación arbórea, suponen sus principales características físicas (Vaca Lorenzo, A., 1992) y ejercen una potente influencia sobre su arquitectura tradicional.

\section{LA DIMENSIÓN AMBIENTAL}

Si bien no es posible afirmar que existiera una preocupación ambiental, en el sentido contemporáneo, detrás de las actuaciones constructivas tradicionales, la escasez material y energética de las sociedades rurales favorecía la contemplación respetuosa del entorno como fuente de numerosos recursos, imprescindibles para la subsistencia. Esta actitud, unida a las limitaciones tecnológicas existentes, impulsaba una explotación sensata y cuidadosa del medio, evitando superar su capacidad ecológica de regeneración, para no poner en peligro la forma de vida conocida. Se trata de la misma visión que está detrás de las actuales teorías del desarrollo sostenible, que proponen volver a recuperar una relación de equilibrio entre el ser humano y el entorno. Para lograr este equilibrio, existen diversas herramientas que se pueden aplicar en el sector de la construcción, como la valoración de los diversos impactos ambientales a través del estudio del ciclo de vida ${ }^{6}$. Se analizan todas las fases que intervienen en el proceso de la edificación: la extracción de recursos y posterior fabricación de materiales, la ejecución de la obra, el uso y mantenimiento por parte de sus habitantes y, finalmente, su demolición y la eliminación de los residuos. Una aproximación análoga, aplicada al caso concreto de la arquitectura popular de Tierra de Campos, puede aclarar su validez como ejemplo de edificación ecológica.

El proceso productivo comienza con la explotación de las materias primas necesarias, principalmente el agua, captada a través de pozos que explotan los acuíferos subterráneos, y los materiales de construcción. Las propiedades del medio físico ejercen de condicionantes que disminuyen las opciones existentes: la disponibilidad y proximidad de los recursos se impone como factor decisivo, teniendo en cuenta la dificultad y el coste del transporte dentro de una sociedad rural tradicional, aislada geográficamente y atrasada en cuanto a avances tecnológicos. Esto conduce a que se empleen materiales locales (sobre todo arcilla y cal), trabajados de forma elemental y sin ser apenas transformados, debido a las restricciones energéticas existentes. Al

\footnotetext{
${ }^{6}$ El Ciclo de Vida es el conjunto de etapas sucesivas que van asociadas a la producción de bienes y servicios, su estudio sistemático y científico, dentro del sector de la edificación, sirve para evaluar los impactos ambientales de una construcción concreta.
} 
provenir estos materiales del propio subsuelo de la parcela en cuestión o de sus cercanías no se produce apenas gasto energético debido al acarreo.

Figura 1. Vista típica del paisaje de Tierra de Campos, en las cercanías de Valderas.

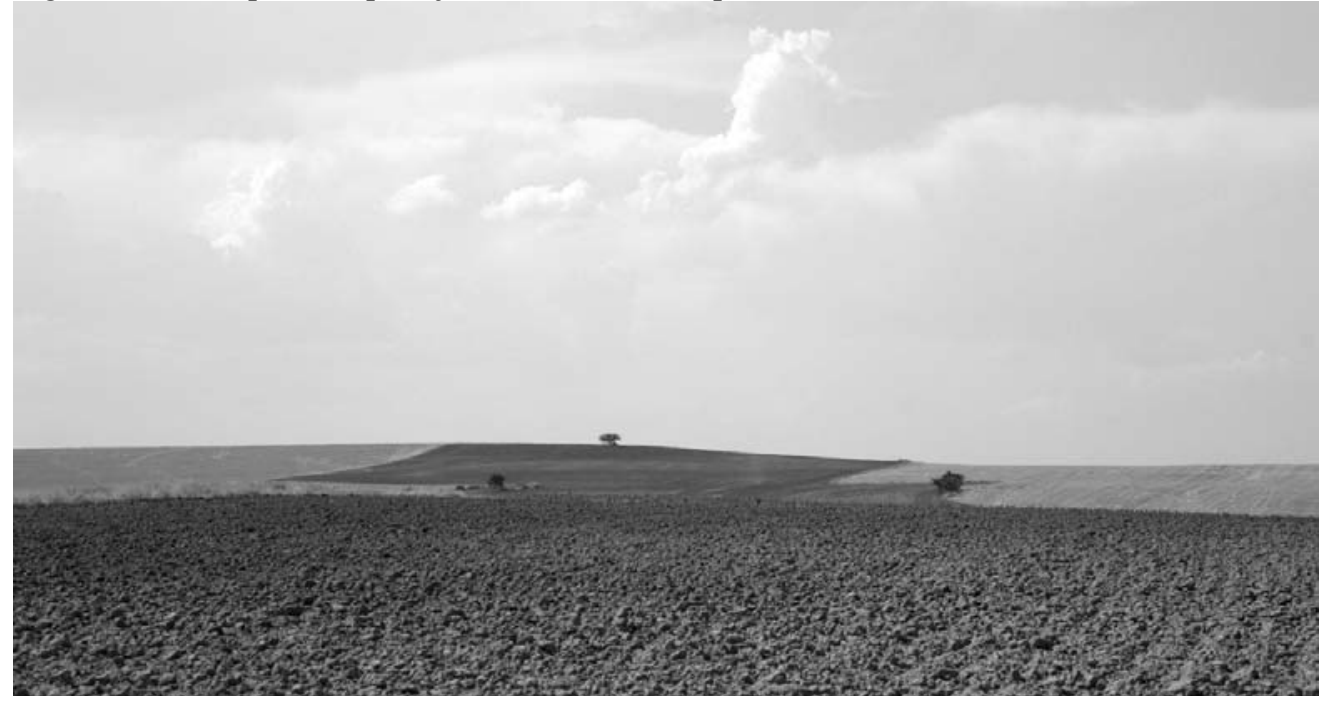

Fuente: Elaboración propia.

Teniendo en cuenta que no existían grandes recursos forestales, no era viable la adopción de sistemas constructivos basadas en la madera, como los entramados existentes en las provincias vecinas de León o Burgos, quedando limitado su uso a los elementos lineales imprescindibles para la construcción de los forjados y las cubiertas. La propia ausencia de biomasa de tipo leñoso limitaba en gran medida la fabricación de ladrillo, que requería de un alto consumo energético, solamente asumible para las edificaciones más representativas. En la comarca escaseaba la piedra, por lo cual solamente se empleaba para la construcción de edificios singulares, como templos o castillos, que representaban a las instituciones más poderosas. Por lo tanto, el constructor popular contaba con un único material disponible de forma inmediata: el suelo arcilloso de color ocre bajo sus propios pies (García Mercadal, 1930), que se empleaba en crudo, con el único aporte energético de la radiación solar incidente durante el proceso de secado.

Esta limitación material, que aparentemente determina un empobrecimiento formal y una merma de las posibilidades constructivas, por el contrario, gracias al ingenio y a la habilidad de los artesanos locales, se convierte en el principal atractivo de su arquitectura popular. La generalización de una serie de sistemas constructivos basados en el empleo del "barro crudo", es decir, sin cocción en horno, caracteriza en gran medida todo el patrimonio construido y le proporciona esa homogeneidad que -a ojos del observador contemporáneo- lo hace tan atractivo, por su perfecta integración en el entorno, desde el punto de vista visual y energético. 
Figura 2. Vista de los tejados de Cuenca de Campos.

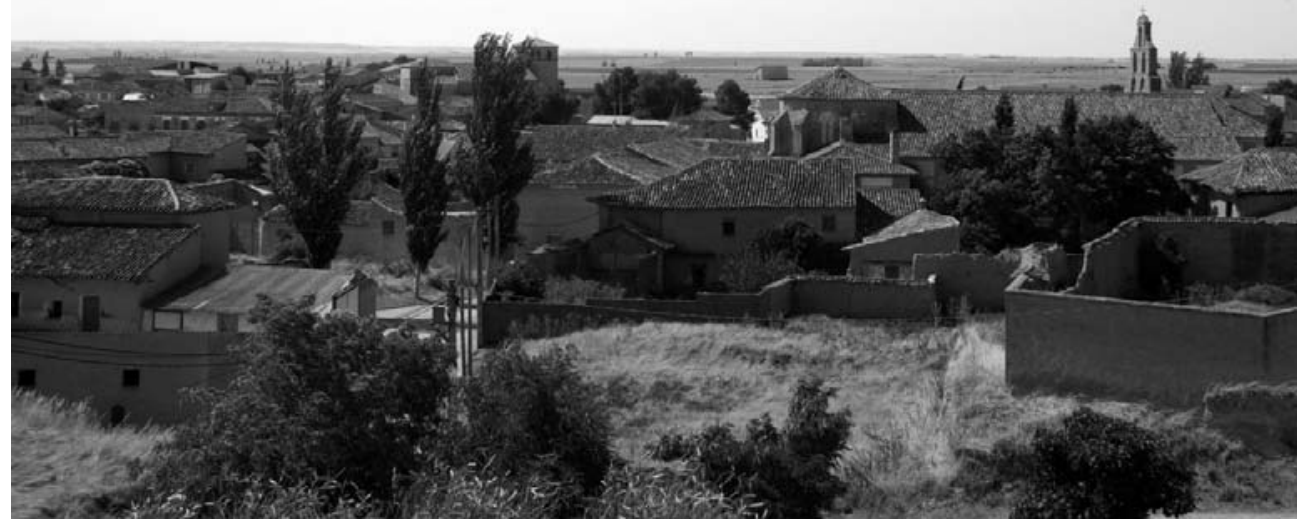

Fuente: Elaboración propia.

En efecto, como consecuencia de la dificultad de levantar estructuras ligeras, por falta de un material propicio, se recurre a la construcción mediante muros de carga que asumen la labor portante gracias a su gran espesor, que suele ser de entre $45 \mathrm{y}$ $60 \mathrm{~cm}$. La masividad de los muros de tapial o adobe viene además impuesta por la fragilidad del propio material, muy sensible a los agentes meteorológicos como el viento y la lluvia. Pero esta aparente desventaja estructural no es tal, en términos globales, ya que se ve compensada durante la vida útil del edificio por las propiedades bioclimáticas de los muros, en concreto su gran aislamiento e inercia térmica.

El aislamiento térmico, conseguido gracias al espesor de los muros y reforzado en el caso del adobe por la mezcla de barro y briznas de paja, creando pequeñas "cámaras de aire", permite limitar pérdidas térmicas en la estación fría y reducir así el consumo energético de la vivienda. A esto también contribuye la introducción de un eficiente sistema de calefacción, de probable origen romano (Torres Balbás, L., 1934), como es la "gloria castellana". Está formado por una serie de conductos bajo el suelo cerámico, que permiten el flujo de aire caliente proveniente de la hoguera, abastecida por los restos de rastrojo, recurso renovable y fácilmente disponible gracias a la gran extensión de cultivos de cereales en la comarca. De esta manera, se consigue el acondicionamiento interior sin producirse la quema de combustibles fósiles y el balance global de emisiones se considera nulo, teniendo en cuenta que la combustión de biomasa devuelve a la atmósfera el $\mathrm{CO}_{2}$ absorbido previamente por el organismo vegetal durante la fotosíntesis.

Además de lo anterior, no hay que olvidar la inercia térmica, propiedad clave para conseguir el confort ambiental interior en un clima extremo como el de Tierra de Campos, con grandes oscilaciones de temperatura entre el día y la noche, sobre todo 
en la época calurosa. Los gruesos muros de fachada absorben progresivamente la radiación solar que incide sobre ellos durante el día, cediéndola posteriormente por ambas caras con un cierto desfase temporal. Esto sucede cuando las temperaturas externas son significativamente más bajas, permitiendo evitar la acumulación de calor en el interior de la vivienda (Esteban Sáiz, J.L., 1986).

Por último, destacar el carácter utilitario de la arquitectura popular, que permite su transformación progresiva para adecuarse a las necesidades de sus habitantes, en forma de crecimiento a través de unidades suplementarias o bien mediante la reutilización de sus elementos constructivos para subsiguientes obras. De esta forma, se prolonga casi indefinidamente la vida útil de los edificios y se evita en gran medida la producción de residuos de la construcción.

\section{LA DIMENSIÓN ECONÓMICA}

Los aspectos ambientales y económicos de la arquitectura popular están íntimamente relacionadas, teniendo en cuenta que los recursos ecológicos suponían la principal fuente de riqueza de las sociedades agrarias pre-industriales. Se trata de economías autosuficientes, debido a su aislamiento del exterior, y la localización geográfica es un factor determinante en su desarrollo. En el caso de Tierra de Campos su alejamiento de los centros regionales políticos y comerciales, además de la dureza de su medio físico, que causaba unas precarias condiciones de vida, marcan profundamente el carácter del territorio. Esto se traduce, a nivel arquitectónico, en una carencia de recursos, tanto naturales como humanos, que explica la importancia del concepto de eficiencia, entendida como la máxima optimización de los medios productivos a fin de satisfacer las necesidades existentes.

Si a la hora de estudiar la viabilidad económica de un edificio contemporáneo, como consecuencia de la separación moderna de envolvente y estructura ${ }^{7}$, se cuantifican de forma diferenciada dos variables -energía y materia ${ }^{8}$ - en el caso de la arquitectura popular no es posible disociarlas con nitidez. A nivel energético, como se ha visto anteriormente, la estrategia principal consiste en aprovechar al máximo las fuentes locales, sobre todo aquellas de tipo renovable, como el sol y el viento, reduciendo la dependencia de recursos exógenos, más costosos debido a su lejanía y dificultad de extracción. A nivel material se busca economizar el proceso constructivo a través de la simplificación tecnológica y la reducción de componentes, a la vez que esto permite su ejecución por parte de trabajadores no cualificados, generalmente el propio usuario final, aspecto que abarata el coste final de la obra.

Por lo tanto, se desarrolla un único sistema constructivo y estructural, que resuelve conjuntamente todas las demandas de habitabilidad, desde la creación de espacios

\footnotetext{
${ }^{7}$ Uno de Los cinco puntos de una nueva arquitectura, presentados por Le Corbusier en 1926, es la fachada libre, lograda a través del retroceso de los pilares respecto del cerramiento exterior.

${ }^{8}$ Como demuestra la habitual división técnica entre el cálculo de instalaciones y de estructuras, realizada incluso por diferentes equipos de especialistas en ingeniería.
} 
bien dimensionados y útiles, hasta el logro de un adecuado confort higrotérmico, a un coste energético razonable. El instrumento que permite alcanzar este doble objetivo es el sistema de muros de carga, ejemplo de eficiencia en cuanto que resuelve con un solo elemento todos problemas básicos de la edificación, como la resistencia portante, la impermeabilización interior o el aislamiento térmico. En Tierra de Campos coexisten dos sistemas murarios que comparten la misma materia prima, el barro, pero que, sin embargo, responden a distintas lógicas constructivas.

En primer lugar tendríamos el tapial, que supone una versión económica y simplificada del sistema de muros ejecutados "in situ" por los romanos, denominado opus caementicium ${ }^{9}$. La variante popular prescinde de los acabados exteriores nobles, con el propósito de economizar, empleando un encofrado desmontable de tablones de madera, que se hace avanzar vertical- y lateralmente hasta completar el muro. A medida que se levanta la tapia mediante módulos de una altura de entre 80 y $90 \mathrm{~cm}$, se compactan las sucesivas tongadas con un pisón, produciendo la suficiente cohesión entre las partículas de arcilla para que el muro resista las cargas (Alonso Ponga, J. L., 1986).

Figura 3. Muros de tapial y adobe, en Aguilar de Campos.

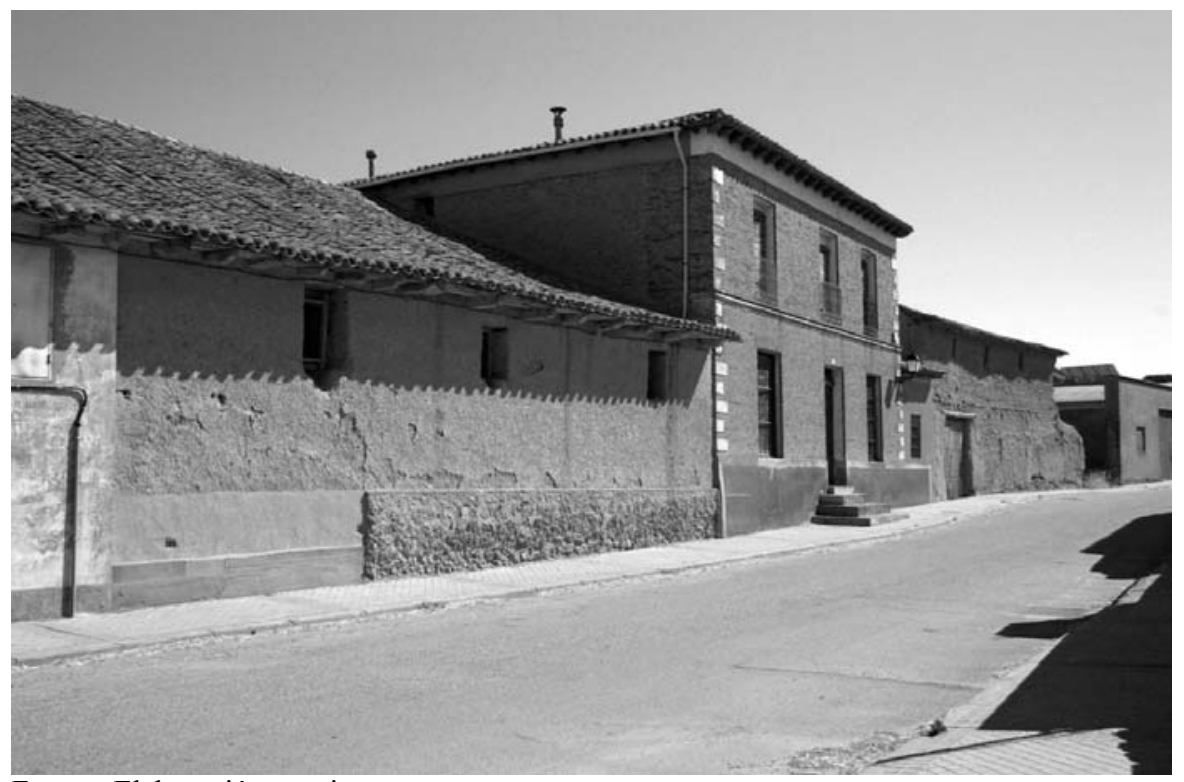

Fuente: Elaboración propia.

\footnotetext{
${ }^{9}$ Muro ejecutado "in situ" compuesto por dos hojas paralelas de fábrica de piedra o ladrillo, que se rellenaban mediante piedras ligeras y cascotes, conglomeradas por una argamasa de cal y arena.
} 
Como su propio nombre indica, se emplea este sistema para la construcción de todo tipo de tapias para corrales y patios, además de los muros más gruesos de las edificaciones, que corresponden a las plantas inferiores. El criterio de eficiencia estructural llevaría a optimizar el grosor de los muros dimensionándolos según las cargas verticales que deban soportar y, en consecuencia, los muros superiores, menos solicitados, deberían ser más esbeltos. Pero este menor espesor de tapial implica una desventaja energética -la pérdida de capacidad aislante-, que unida a su gran peso propio, que dificulta su puesta en obra a varios metros de altura, y aconseja la introducción de una nueva técnica constructiva. Se trata del adobe, que aúna ligereza y aislamiento térmico, y constituye un sistema económico, que permite aligerar los muros superiores.

El adobe obedece, pues, a otra lógica constructiva, y tiene una estrecha relación con el ladrillo, hasta el punto de que se podría considerar su precedente histórico, en épocas donde la escasez energética era la norma. Ambas técnicas responden a una voluntad de prefabricación para conseguir un elemento más manejable y ligero que permita una rápida y cómoda puesta en obra. La principal diferencia entre el adobe y el ladrillo, como se apuntado anteriormente, es la ausencia de cocción del primero, que propicia otra diferencia crucial, como es el añadido de un material fibroso como la paja trillada, que frena su tendencia a resquebrajarse por retracción y le aporta mayor resistencia mecánica a flexión, compensando su menor cohesión interior. Esta característica constructiva es también clave en mejorar el comportamiento térmico del material, ya que las briznas de paja funcionan a modo de pequeñas "cámaras de aire", que tienen una gran capacidad aislante.

Existen diferentes tamaños de adobe, en función de su posterior finalidad constructiva, ya sea la ejecución de muros de fachada o tabiques interiores, más esbeltos que los anteriores. La mezcla de arcilla, limo y arena es la que reúne mejores propiedades para el adobe y tras ser humedecida y mezclada con la paja se introduce en el molde o "adobera" que le da forma de prisma antes de su compactación. La retirada del molde es necesaria para la aireación por los cuatro lados y el secado lento al sol, que le termina de dotar de sus propiedades finales (Rohmer, E., 1985). La ejecución de los muros se produce mediante la colocación de hiladas horizontales donde la adherencia entre las piezas se consigue mediante el uso de barro. Finalmente, se terminan los muros con un revoque que sella las juntas entre los adobes, para evitar filtraciones, que podrían comprometer su durabilidad.

La variante más singular de revestimiento en la comarca es el "trullado", que otorga a sus edificios de un acabado exterior característico, debido a la particular textura conseguida por la mezcla de barro y briznas de paja, que evita el cuarteamiento del acabado por la acción del sol o la lluvia. Este acabado aparece aplicado tanto en muros de tapial como de adobe, haciéndolos difícilmente distinguibles a simple vista y consiguiendo una apariencia exterior altamente homogénea. La fragilidad del barro frente al agua hace necesario su cuidado periódico y la reposición de los revocos cada pocos años, siendo éste uno de los principales problemas de su mantenimiento, introduciendo además un coste añadido. 


\section{LA DIMENSIÓN CULTURAL}

A la hora de valorar la sostenibilidad de un proyecto urbano o edificatorio no se han tenido suficientemente en cuenta, hasta el momento, los aspectos culturales. Sin embargo, en el actual contexto de globalización económica que impulsa rápidamente las tendencias artísticas y tecnológicas a través de las fronteras geográficas, se hace necesario reflexionar sobre la idoneidad de implantar, de forma indiscriminada, modelos arquitectónicos pretendidamente universales dentro de un contexto local. La constatación de los fuertes impactos negativos, que se han producido en el entorno construido, como la pérdida de identidad espacial (Augé, M., 1993) o la degradación del paisaje tradicional (Muñoz, F., 2008), contrasta con una visión de la arquitectura popular como materialización de unos determinados valores culturales, sociales y ambientales, que caracterizan a una comunidad. Desde el punto de vista cultural existen dos posibles aproximaciones a la misma: por una parte, el estudio de las edificaciones como fenómenos aislados y, por otra, el análisis de sus interacciones con el medio natural, formando conjuntamente el paisaje cultural.

La unidad arquitectónica y paisajística lograda por las construcciones populares de Tierra Campos, independientemente de su función, tamaño o morfología, se debe a la absoluta predominancia del barro como material de construcción. Se trata de unos conjuntos edificados altamente homogéneos, que se integran perfectamente en el entorno, gracias a la coincidencia cromática y de texturas. Esto contribuye, junto con otros elementos ajenos a la arquitectura, como la gastronomía o el folclore, a crear una identidad cultural propia y diferenciada del exterior, que refuerza el sentido de pertenencia al colectivo de sus habitantes (Sotelo, J. A., 1992), a la vez que muestra "la manera en que desea gestionar su relación existencial con las particularidades del lugar" (Almeida, C. ; Mora, J. y Dos Reis, F., 2010).

Figura 4. Alzado del núcleo de población de Barcial de la Loma.

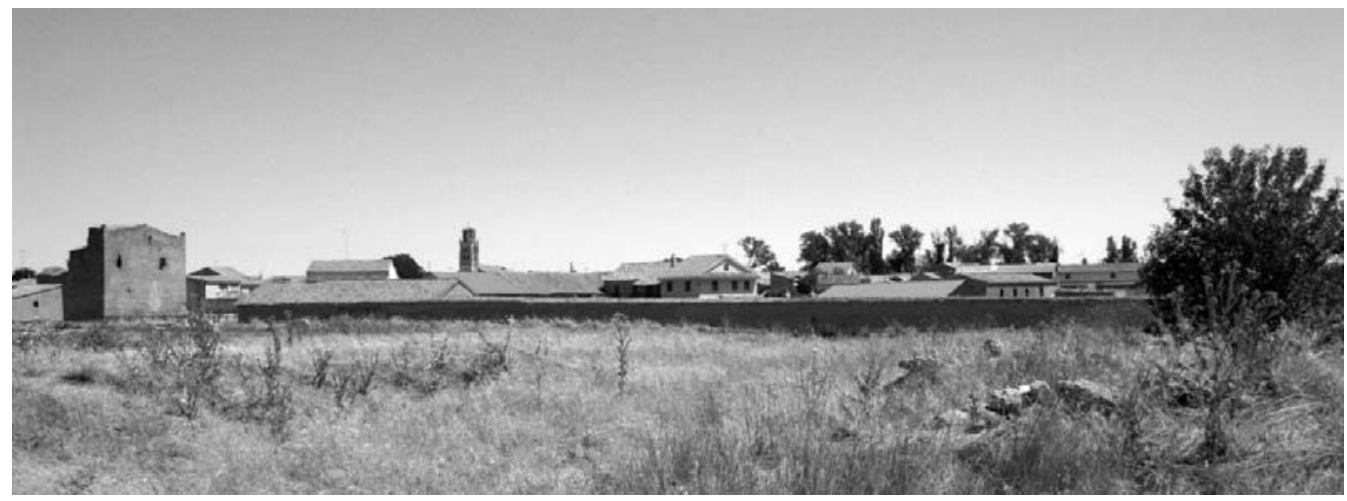

Fuente: Elaboración propia. 
Esta simbiosis entre arquitectura y paisaje se explica a través de varios factores, destacando entre ellos el origen dual de la edificación popular, como elemento residencial y unidad mínima de producción, que favorece una implantación "orgánica" en el medio. En efecto, su propia morfología y la forma de agrupación de sus elementos (vivienda, patios, construcciones auxiliares, etc.), ignoran las reglas compositivas de la arquitectura clásica ${ }^{10}$, no encontrándose generalmente ejes de simetría o repeticiones seriadas de elementos, como en el caso de los órdenes arquitectónicos grecoromanos. Se generan, de esta forma, conjuntos de apariencia desordenada y espontánea que se adaptan a las lógicas estructurales y perceptivas existentes en la naturaleza, logrando una perfecta integración en el paisaje, como transmiten de forma expresiva los profesores Padilla y Del Arco: “...la casa se mimetiza a menudo en el entorno en que se inscribe, se une al paisaje, adoptando las formas naturales que la rodean; casas altas, puntiagudas, en las montañas; bajas y como aplastadas en las llanuras" y más adelante "Esa sensación íntima de edificio vivo, activo y personal es la que nos provoca la idea de coherencia, de armonía, en la observación del paisaje popular tradicional, o de la buena arquitectura rural, donde el hombre y la naturaleza son interactivos" (Del Arco, E. y Padilla, C., 1990).

Además de lo anterior, es necesario referirse a la importancia de los conocimientos transmitidos de forma oral a través del tiempo (técnicas constructivas y estrategias proyectuales), que de generación en generación sirvieron para perfeccionar las construcciones populares, descartando las opciones que resultaban poco funcionales y antieconómicas. Este proceso, análogo al concepto de selección natural ${ }^{11}$ de los seres vivos en la teoría de la evolución, desemboca en la aparición de unos modelos recurrentes, que se sintetizan, a efectos prácticos, a través del concepto de tipo edificatorio (Sáinz Guerra, J. L. y otros, 2012).

El tipo más común en Tierra de Campos es la casa de labranza, que se compone de dos zonas espaciales diferenciadas, de acuerdo con su función predominantemente residencial o agrícola. La vivienda, que se sitúa en el frente edificado hacia la calle, es de pequeño tamaño -entre 120 y $250 \mathrm{~m}^{2}$ - y no supera las dos plantas de altura, culminadas por una cubierta inclinada con dos vertientes. La planta baja alberga los espacios comunes como el zaguán, la cocina, la sala con gloria y, por lo general, las alcobas. En el caso de viviendas de mayor entidad estas alcobas pasan a la planta primera, mientras que el espacio de bajocubierta aloja el granero.

\footnotetext{
${ }^{10}$ En el sentido de la tradición arquitectónica clásica, transmitida a través de los tratados que difunden el estilo griego y romano, sobre todo a partir del Renacimiento.

${ }^{11}$ Es el proceso a través del cual, los organismos mejor adaptados desplazan a los menos adaptados mediante la acumulación lenta de cambios genéticos favorables en la población a lo largo de las generaciones.
} 
Figura 5. Plantas de casa de labranza en Aguilar de Campos.
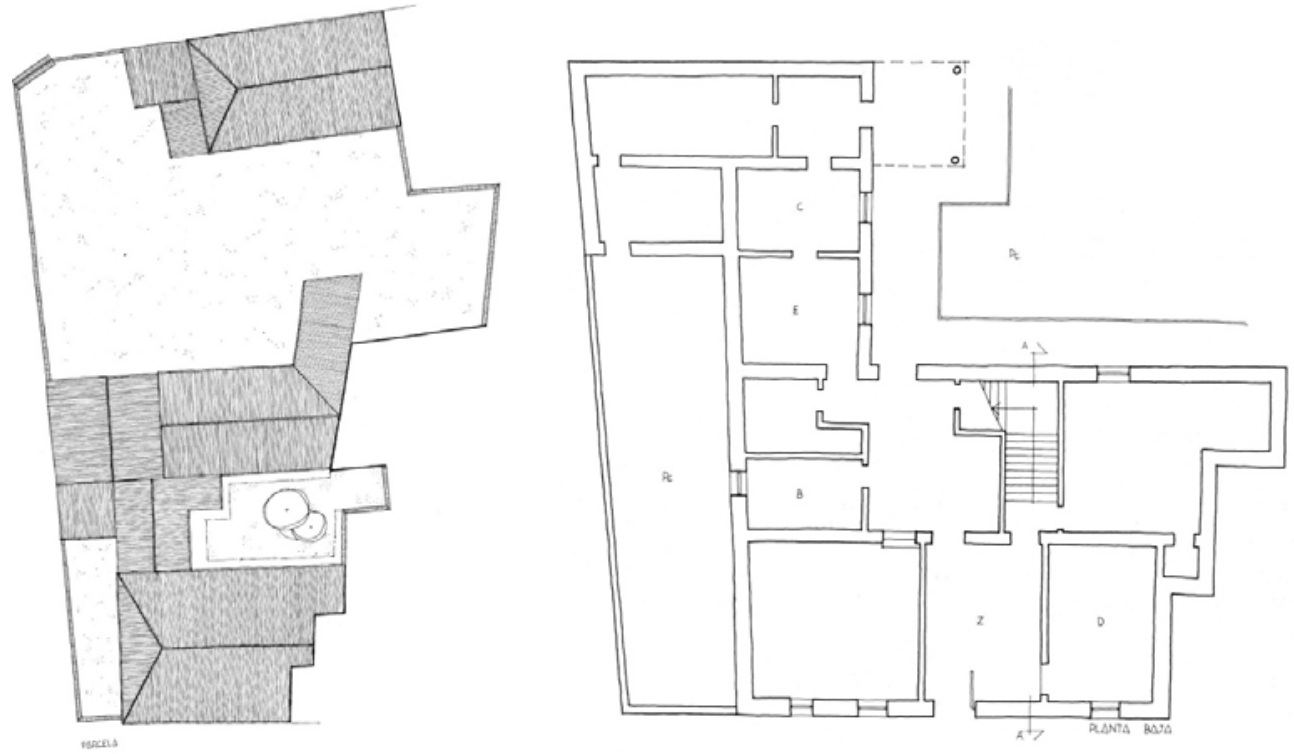

Fuente: Moya, L. y Otros (1986).

Este conjunto edificado se completa con un "corral" o espacio abierto trasero, alrededor del cual se organizan todas las construcciones auxiliares, como las cuadras, los casetos (cochiqueras), los pajares y las lastras. En este espacio se encuentra también la cocina de matanza o de verano, con el horno incorporado y adosado a la edificación ${ }^{12}$. Se accede al corral tanto desde la propia vivienda como desde la calle, a través de unos grandes portones de madera.

Frente a los edificios residenciales, citados anteriormente, existen también otros tipos arquitectónicos de gran interés cultural, testigos de una forma de vida rural, hoy en día prácticamente desaparecida. Se trata de las singulares construcciones agrarias de Tierra de Campos, como palomares, bodegas y chozos de pastor, que se originaron para satisfacer las necesidades productivas de sus habitantes.

\footnotetext{
${ }^{12}$ Para lograr un aprovechamiento térmico interior del mismo, además de economizar su construcción, compartiendo materiales con la vivienda.
} 
Si bien la historia del palomar proviene probablemente de la tradición romana del cuidado y explotación de las palomas, no es hasta finales del s.XVIII cuando se desarrolla una verdadera cultura del mismo, como complemento a las labores propias del campo. Las condiciones ecológicas del territorio, como la deforestación, que reducía la presencia de caza mayor, o la ausencia de pastos, que limitaba la ganadería, provocaban cierta escasez de proteínas en la dieta campesina. Aparece entonces el recurso de los pichones, que pasa a ser un alimento muy preciado por su alto valor gastronómico. El palomar es además de utilidad para la agricultura gracias al estiércol que en él se podía recoger y que servía como abono para los campos. Por estos motivos se difunde el palomar por toda Tierra de Campos, como una edificación indispensable por su valor económico y social, y llega a constituirse en el símbolo construido del territorio, gracias a su singularidad plástica, que contrasta con el anonimato y la austeridad del resto de la arquitectura popular.

Los palomares se encuentran distribuidos por toda la comarca, ya sea de forma aislada o formando conjuntos, y en ocasiones se ven cercados por una tapia, que delimita un pequeño recinto. Generalmente, no aparecen demasiado alejados de los núcleos de población por motivos de conveniencia y para poder ejercer cierto control sobre su acceso. Los tipos más habituales son los redondos y cuadrados, aunque en ocasiones se encuentran también palomares rectangulares, poligonales o mixtos. Dependiendo de su tamaño pueden incluir un patio interior y otra característica singular es, en algunos casos, el escalonamiento progresivo de los tejados, formando una estructura piramidal. Resulta sorprendente la variedad de tipos de palomar, existiendo varias hipótesis que explican su exuberancia formal. Una de las más plausibles es la voluntad de originalidad y diferenciación de sus propietarios, teniendo en cuenta que la posesión de uno o más palomares era un símbolo de opulencia que otorgaba cierto prestigio dentro de las cerradas comunidades rurales (Roldán Morales, F. P., 1983).

Figura 6. Palomar en Villavicencio de los Caballeros.

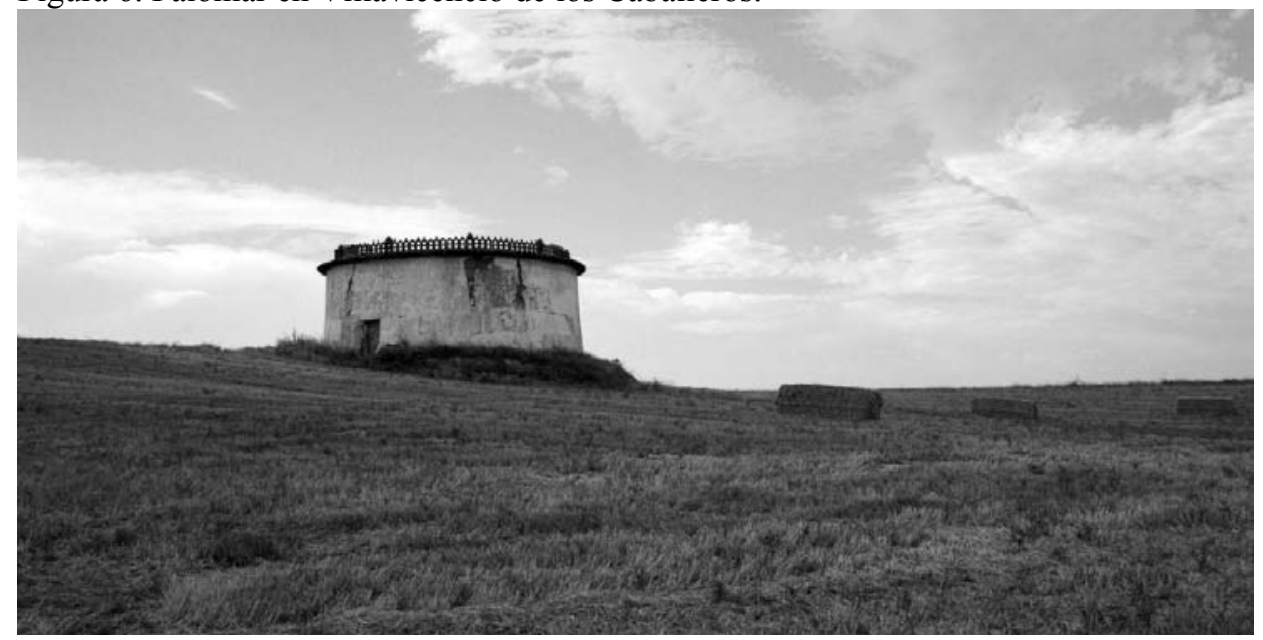

Fuente: Elaboración propia. 
Otro importante ejemplo de arquitectura popular son las cuevas o bodegas, construcciones enterradas para la elaboración y el almacenaje del vino, gracias a la facilidad para excavar el terreno arcilloso. Gracias al excepcional aislamiento térmico que proporciona el abrigo del terreno, se crea un micro-clima ideal en su interior con temperaturas relativamente constantes a lo largo del año, en torno a los $15^{\circ}$, y que permiten la óptima elaboración y conservación del vino. Estas bodegas rupestres son espacios excavados en pequeñas lomas o promontorios cercanos a los pueblos, aprovechando el desnivel y formando complejos con estrechas galerías subterráneas, que conectan a su vez espacios más amplios.

Las bodegas, junto con el resto de construcciones agrarias auxiliares como las casetas, los chozos y los colmenares, hoy en día abandonados, forman un patrimonio de gran valor que puebla los alrededores de los núcleos de población y los campos. Su valor etnográfico y antropológico, además de su variedad formal, en ocasiones llegando a generar formas orgánicas e inesperadas, aportan un contrapunto estético que enriquece el paisaje de la comarca.

Figura 7. Bodegas en Herrín de Campos.

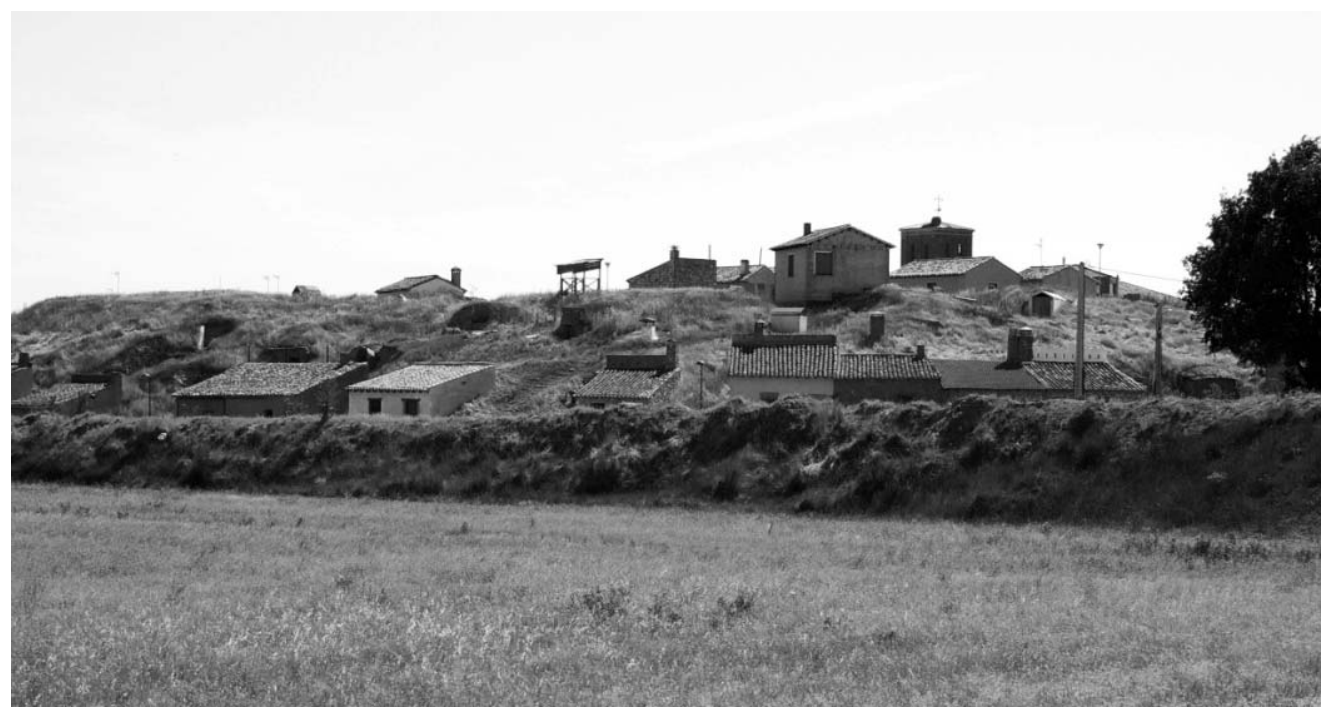

Fuente: Elaboración propia. 


\section{LA DIMENSIÓN SOCIAL}

Los conjuntos de arquitectura popular, que forman el tejido edificatorio de los núcleos de población de Tierra de Campos, tienen la doble función de permitir el desarrollo de las actividades individuales, a la vez que crean un marco espacial para la vida colectiva. Estos aspectos sociales se ponen de manifiesto al repasar la historia de la comarca, que dio lugar a su hábitat característico.

Figura 8. Plano de núcleo de población e inmediaciones, en Becilla de Valderaduey.

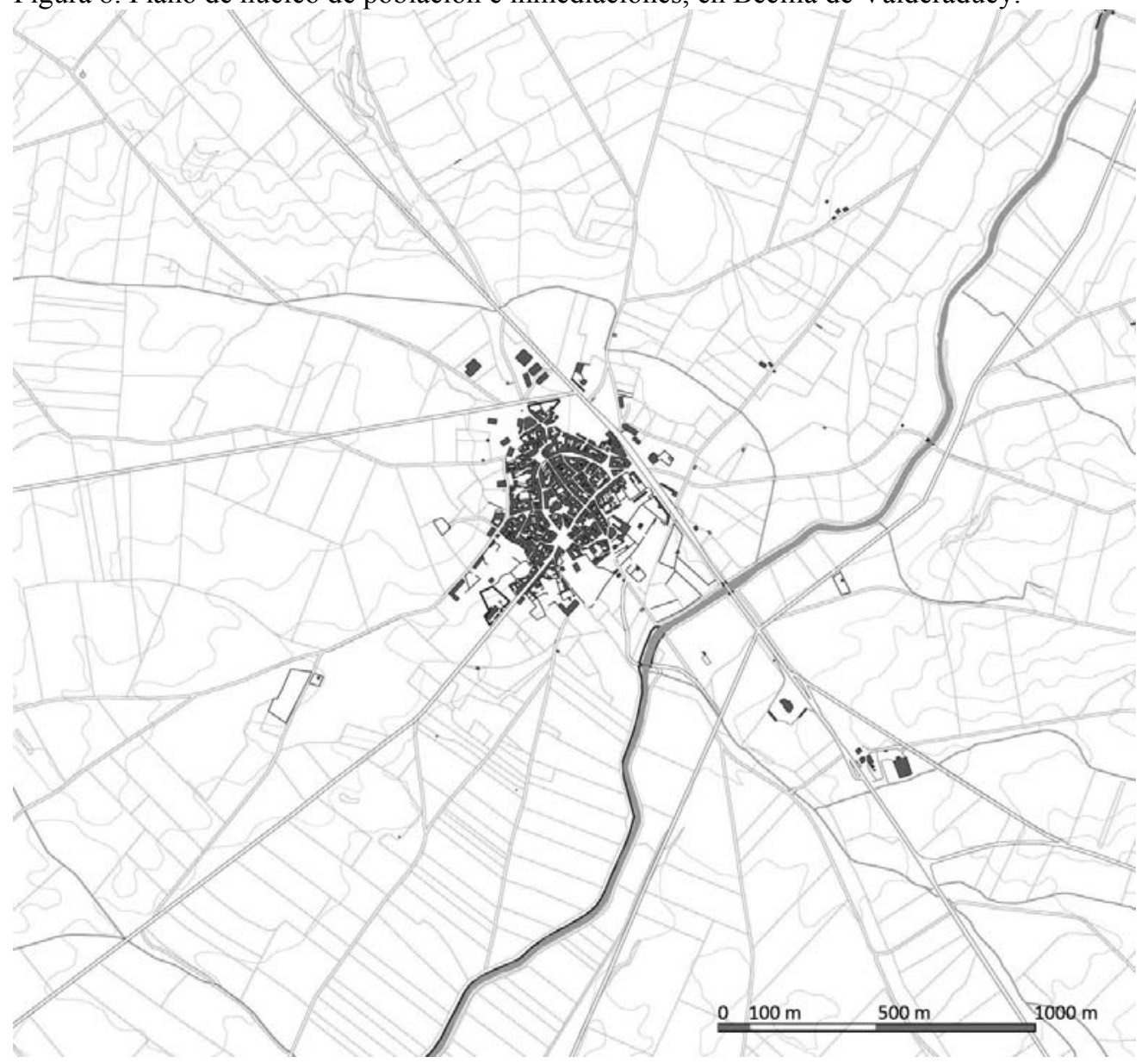

Fuente: Elaboración propia, a partir de la cartografía digital de la Dirección General del Catastro. 
Los primeros pobladores de la región, según las fuentes arqueológicas, fueron los vacceos, pueblo céltico prerrománico que practicaba una agricultura de tipo colectivista. La explotación común del terrazgo, mediante la roturación colectiva o dirigida, genera el sistema conocido como openfields o campos abiertos ${ }^{13,}$ que, según el geógrafo André Meynier, favorece la agrupación de la población en núcleos compactos y separados entre sí. Este hábitat concentrado es un modelo que se traslada hasta nuestros días, ya que permite el máximo aprovechamiento productivo del territorio y se ve favorecido por la uniformidad de las condiciones geográficas y ecológicas de la comarca. Las condiciones típicas del poblamiento son la existencia de un terrazgo con una superficie aproximada de 25 a $30 \mathrm{~km}^{2}$ por cada núcleo, que se sitúa de forma central respecto del mismo, y una distancia media entre pueblos vecinos de unos 5 a 10 km (Benito Martín, F., 1998).

Posteriormente, las circunstancias históricas medievales y, en particular, el proceso de repoblación del territorio al norte del río Duero, asociado a la Reconquista, determinan en gran medida las condiciones urbanas de los asentamientos de Tierra de Campos y, aún hoy, son claramente perceptibles (Benito Martín, F., 2000). La rápida desintegración del sistema territorial romano-visigótico, después de la invasión árabe que tiene lugar en el s. VIII, provoca la despoblación de la región y su posterior conversión en un área fronteriza altamente inestable. La estructura del territorio no se vuelve a consolidar hasta la Alta Edad Media cuando los reinos cristianos aprovechan la oportunidad de afianzar su dominio militar a través de la repoblación definitiva del territorio. Si bien en un primer momento histórico, durante los siglos IX y X, se produce un modelo de asentamiento que genera una malla relativamente isótropa de pequeñas unidades de explotación agrícola, de similar tamaño e importancia dentro de la trama espacial, este sistema se jerarquiza progresivamente, hasta que en el siglo XII toma forma la estructura actual, donde cada núcleo asume un papel definido.

El nuevo sistema social y económico que se instauró durante la Reconquista generaba otras necesidades, que se materializaron a través de una distinta organización y morfología de los núcleos, ajena a las preexistencias romanas y visigóticas. En el caso de Tierra de Campos se puede observar un tipo predominante, altamente compacto y de forma irregular, denominado como "riñon" o "alubia". Esta forma orgánica se desarrolla debido a una serie de factores, principalmente, la agrupación densa de viviendas tradicionales debido a la antigua presencia de murallas o cercas defensivas en la mayoría de los asentamientos, al tratarse de una zona en conflicto por su condición fronteriza. La forma ideal de círculo, formada por anillos concéntricos alrededor de la iglesia o el castillo, no aparece casi nunca debido a otros factores secundarios, que deforman la trama. En primer lugar, está la topografía, que obliga a una adaptación del tejido a los cerros o a las laderas. En segundo lugar, la presencia de un ele-

\footnotetext{
${ }^{13}$ En oposición a los campos cercados con árboles, setos o muros, típicos de la Europa atlántica. El ejemplo prototípico sería el bocage presente en las regiones francesas de Normandía y Bretaña.
} 
mento lineal como un río o un camino puede crear un vector de crecimiento a su paso "estirando" el asentamiento y provocando una forma irregular. También existen condicionantes de tipo productivo, como la organización del terrazgo o los recursos ecológicos que se desean explotar, como, por ejemplo, aguas subterráneas para el riego o suelos excepcionalmente fértiles en las vegas de los ríos.

Dentro de esta morfología predominante suele aparecer una "almendra" central, compuesta de pequeñas manzanas edificadas -donde prima la función residencial- y rodeada por una serie de vías circulares. A continuación, se encuentra un segundo tipo de tejido, más disgregado y poroso debido a su carácter agrícola, donde coexiste la vivienda con los pajares y los otros espacios auxiliares. Los caminos de ronda que separan estas manzanas del campo, siguiendo el trazado de las antiguas murallas, definen nítidamente los límites del pueblo (Moya, L. y otros, 1983).

Figura 9. Vista aérea del núcleo de población de Herrín de Campos.

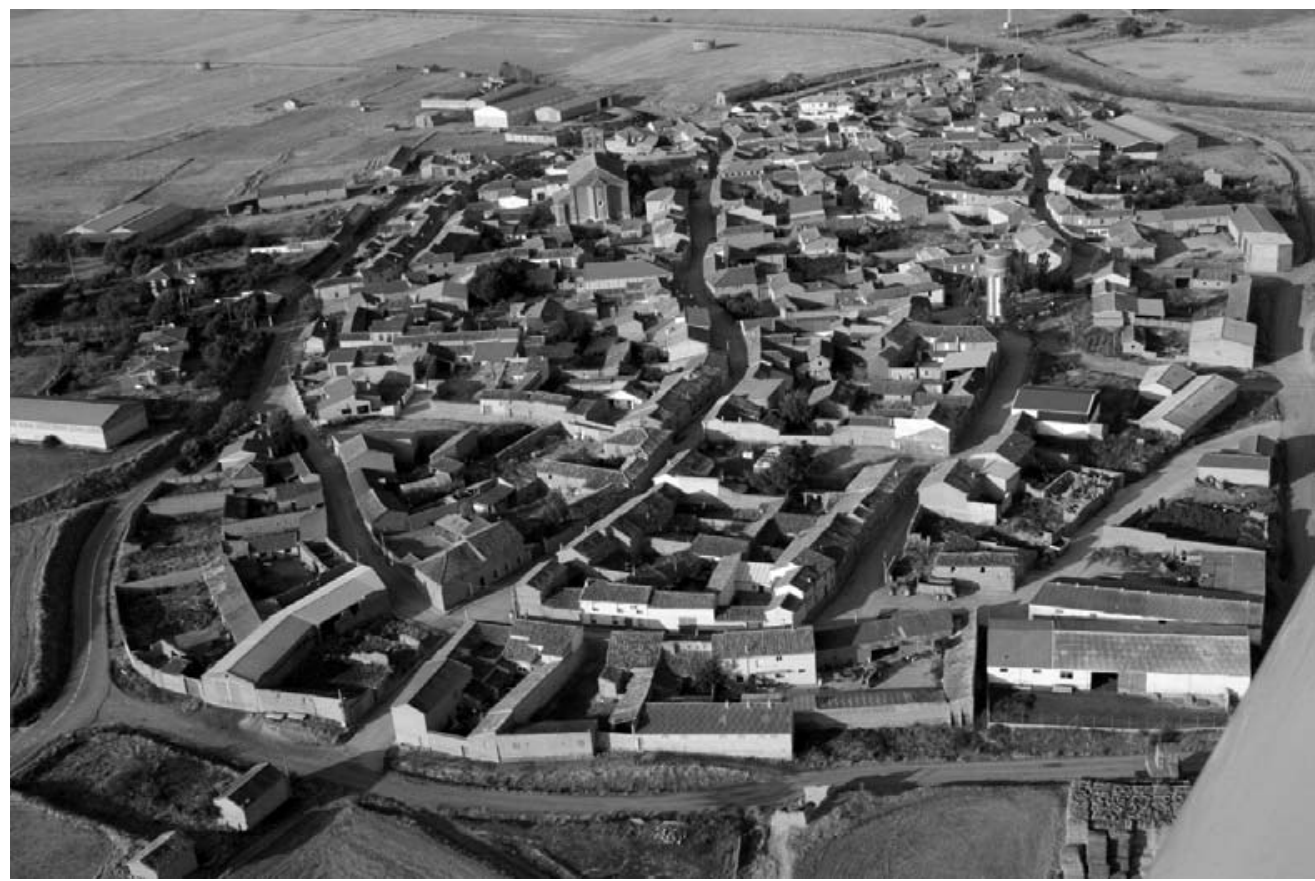

Fuente: Ricardo Melgar.

Los rasgos representativos de los trazados urbanos de la comarca aparecen en núcleos como Becilla de Valderaduey, Herrín de Campos y Villavicencio de los Caballeros. Su superficie total oscila entre las 6 y las 15 Ha y cuentan con calles anchas, largas y rectas o levemente curvadas, que forman entre 10 y 20 manzanas (Benito Martín, F., 1998). Dentro de la estructura viaria destacan los soportales sobre 
pies derechos de madera, formados en ocasiones debajo de las viviendas situadas en las calles más representativas. Estos espacios semi-abiertos facilitaban la convivencia y el tránsito cuando la lluvia transformaba el suelo sin pavimentar en un barrizal, permitiendo el desarrollo de las actividades comerciales. Uno de los espacios urbanos más característicos formados por la arquitectura popular de la comarca es el soportal de la Plaza Mayor de Cuenca de Campos, singular por su tosquedad y autenticidad.

Figura 10. Viviendas sobre soportales en Cuenca de Campos.

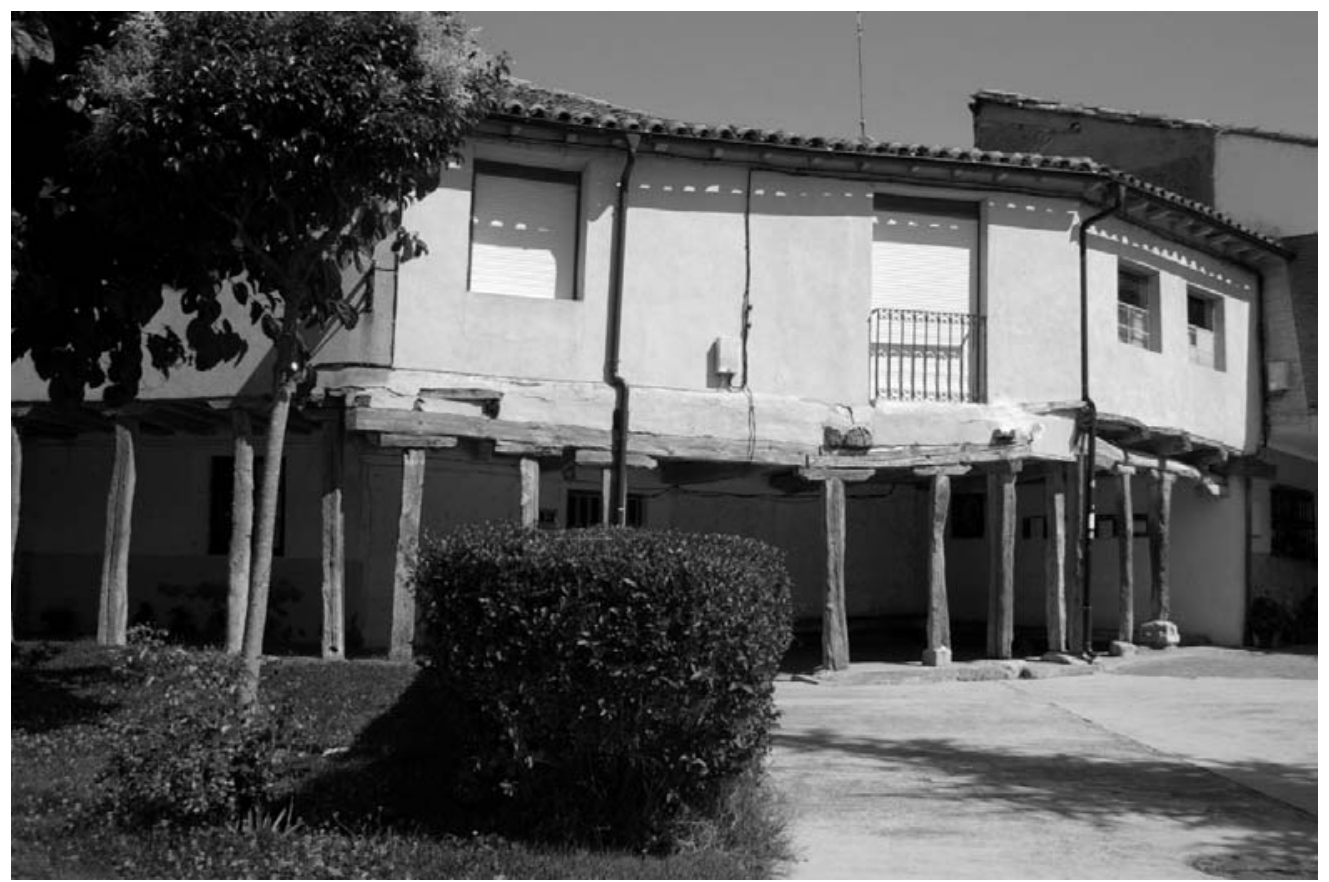

Fuente: Elaboración propia.

La vida colectiva es también posible gracias a la existencia de numerosos espacios públicos abiertos, como plazas y plazoletas. Son generalmente amplias y bien configuradas, pero su forma suele ser irregular y se ubican en el centro del pueblo, no respondiendo a una pauta concreta sino adaptándose a situaciones diversas. Los hitos urbanos, generalmente edificios representativos, como iglesias, conventos o fortificaciones, o bien construcciones funcionales como silos o depósitos de agua, modifican la forma urbana, introduciendo irregularidades dentro de los conjuntos de arquitectura popular, que generan espacios característicos a su alrededor. Se encuentran también numerosos espacios abiertos en el perímetro de los núcleos, junto a las antiguas puertas de las murallas, sirviendo para oxigenar la trama urbana y permitir la visión del paisaje desde el interior de los pueblos. 


\section{CONCLUSIONES}

Los antecedentes al concepto de la sostenibilidad, en el campo de la arquitectura, se encuentran en la construcción bioclimática o ecológica, definida como el conjunto de estrategias de diseño que persiguen la adaptación inteligente del edificio al clima local, minimizando el impacto ambiental producido. El bioclimatismo está inspirado principalmente en la experiencia de la arquitectura popular, emulando ciertos aspectos de la misma en las nuevas edificaciones, como la correcta orientación solar o el aprovechamiento de las corrientes de aire para refrescar el ambiente interior.

El éxito de esta tendencia arquitectónica, unido al indudable prestigio social que va asociado al movimiento ecologista, provocó que se concentraran los esfuerzos en la mejora de los aspectos medioambientales de las edificaciones, descuidando por completo las otras tres dimensiones de la sostenibilidad. La arquitectura popular aparece en este contexto como un ejemplo de gran interés, ya que representa una idea de construcción que se adapta no solamente al medio físico, sino también a la organización social del ser humano, creando una identidad cultural distintiva y, todo ello, utilizando racionalmente los recursos disponibles.

El caso de Tierra de Campos, caracterizado por una integración plena, a todos los niveles, entre arquitectura y entorno, proporciona ciertas claves para avanzar en la búsqueda de un concepto de edificación que favorezca la proximidad de sus habitantes con la naturaleza y que permita la convivencia de los grupos humanos.

Del ejemplo concreto estudiado, se pueden destacar los siguientes aspectos, económica- y medioambientalmente beneficiosos:

- un menor volumen de extracción de materiales del entorno

- ahorro en gastos de transporte y de transformación de los productos

- reducción del consumo energético durante el uso de la vivienda

- adecuada gestión de los recursos hídricos

- rapidez y economía de medios en la ejecución de obra

- flexibilidad funcional y posibilidad de reutilización de los espacios

En los niveles cultural y social se aprecian las siguientes ventajas:

- unidad visual con el medio físico

- creación de una identidad propia de la comunidad

- adaptación a la forma de vida de sus habitantes

- refuerzo de la cohesión social

Todo lo anterior permite concluir que el estudio de las construcciones populares es una de las principales vías para completar la base teórica de la sostenibilidad en arquitectura, conjuntamente con la investigación tecnológica enfocada hacia la integración de las energías renovables en los edificios o la mejora de las propiedades ecológicas de los materiales de construcción, como su capacidad de aislamiento térmico o su mayor durabilidad. 


\section{BIBLIOGRAFÍA}

ALONSO PONGA, J. L. (1986). Huellas de Castilla y León: la arquitectura del barro. Junta de Castilla y León, Valladolid, 1986.

ALMEIDA MARQUES, C.; MORA ALISEDA, J. y DOS REIS CONDESSO, F. (2010). Vivienda y territorio. M+A. Revista Electrónic@ de Medio Ambiente. 2010, 8:1-17.

AUGÉ, M. (1993). Los "no lugares": espacios del anonimato: una antropología de la modernidad. Gedisa, Barcelona.

BENITO MARTÍN, F. (1998). La arquitectura tradicional de Castilla y León. Junta de Castilla y León, Consejería de Medio Ambiente y Ordenación del Territorio, Valladolid.

BENITO MARTÍN, F. (2000). La formación de la ciudad medieval: La red urbana en Castilla y León. Secretariado de Publicaciones e Intercambio, Valladolid.

DEL ARCO MARTÍN, E. Y PADILLA, C. (1990). La arquitectura popular como emblema. Arquitectura popular en España / coord. por Luis Angel Sánchez Gómez, Antonio Cea Gutiérrez, Matilde Fernández Montes. CSIC, Madrid.

ESTEBAN SÁIZ, J. L. (1986). La arquitectura popular como base de una arquitectura bioclimática. Aplicación al enfriamiento pasivo. Informes de la construcción, n³8, pp. 59-69, CSIC, Instituto de Ciencias de la Construcción "Eduardo Torroja", Madrid.

FLORES LÓPEZ, C. (1974). Arquitectura popular española. Volumen $1^{\circ}$. Aguilar, Madrid, 1976.

GARCÍA MERCADAL, F. (1930). La casa popular en España. Espasa Calpe, Madrid, 1981.

GARCÍA MERINO, L. V. (1988). Análisis del Medio Físico de Valladolid. Delimitación de unidades y estructura territorial. Junta de Castilla y León, Valladolid.

MARGALEF, R. (1963). On certain unifying principles in ecology. The American Naturalist, Vol 97, No. 897; pp. 357-374, University of Chicago Press, Chicago. [Traducción propia]

MEYNIER, A. (1968). Los paisajes agrarios. Panoramas de la Geografía Universal n³6. Ediciones Moretón, Bilbao.

MORÁN RODRÍGUEZ, M. A. Y NAVARRO MADRID, A. (1992). La Arquitectura popular como Urbanismo ecológico: el habitat de Zamora, en Anales de Geografía de la Universidad Complutense, $n^{\circ} 12$, pp.243-253, Servicio de Publicaciones, Universidad Complutense, Madrid.

MORÁN RODRÍGUEZ, M. A. (1998). Arquitectura popular y Medio Ambiente. Observatorio Medioambiental, $\mathrm{n}^{\circ} 1$; pp. 287-294, Servicio de Publicaciones, Universidad Complutense, Madrid.

MOYA, L. Y OTROS (1983). Tipologías arquitectónicas y urbanísticas de la provincia de Valladolid. Dto. de Urbanismo de la Escuela de Arquitectura de Valladolid, Valladolid. [Texto inédito mecanografiado] 
MUÑOZ, F. (2008). urBANALización: Paisajes Comunes, Lugares Globales. Gustavo Gili, Barcelona.

OLIVER, P. (1997). Encyclopedia of vernacular architecture of the world. Vol.1, Theories and principles. Cambridge University Press, Cambridge. [Traducción propia]

RAPOPORT, A. (1969). House Form and Culture. Prentice Hall Foundations of Cultural Geography Series, Ed. Philip L. Wagner, New York, 2002. [Traducción propia]

RED MUNDIAL DE CIUDADES, GOBIERNOS LOCALES Y REGIONALES (2010). Culture: Fourth Pillar of Sustainable Development.

REAL ACADEMIA DE LA LENGUA ESPAÑOLA (2013): Diccionario de la lengua española. Vigésima TORRES BALBÁS, L. (1934)segunda edición.

ROHMER, E. (1985). La tierra, material de construcción. Proyecto Navapalos 85, Madrid.

ROLDÁN MORALES, F. P. (1983). Palomares de barro de Tierra de Campos. Caja de Ahorros Provincial de Valladolid, Valladolid.

SÁINZ GUERRA, J.L. Y OTROS (2012). Edificios y conjuntos de la arquitectura popular en Castilla y León. Edición electrónica: Consejería de Cultura y Turismo, Junta de Castilla y León.

SOTELO NAVALPOTRO, J.A. (1991). Paisaje, semiología y análisis geográfico. Anales de Geografía de la Universidad Complutense, $n^{\circ} 11$, pp. 11-23.

SOTELO NAVALPOTRO, J.A. (2000). Problemas ecológicos de la conservación del patrimonio y del Medio Ambiente. Anales de Geografía de la Universidad Complutense, $\mathrm{n}^{\circ} 20$, pp. 193-215.

TORRES BALBÁS, L. (1934). La vivienda popular en España. En Folklore y Costumbres de España, Tomo III, pp. 137-502. Editorial Alberto Martín, Barcelona.

VACA LORENZO, A. (1992). La Tierra de Campos y sus bases ecológicas en el siglo XIV. Studia Histórica. Historia Medieval, nº10, pp. 149-186, Ediciones Universidad de Salamanca, Salamanca. 